\title{
Gamma-ray spectral modulations induced by photon-ALP-dark photon oscillations
}

\author{
Kiwoon Choi, ${ }^{1, *}$ Sangjun Lee, ${ }^{2,1, \dagger}$ Hyeonseok Seong, ${ }^{2, \ddagger}$ and Seokhoon Yun ${ }^{2,1,3, \S}$ \\ ${ }^{1}$ Center for Theoretical Physics of the Universe, Institute for Basic Science, Daejeon 34051, South Korea \\ ${ }^{2}$ Department of Physics, KAIST, Daejeon 34141, South Korea \\ ${ }^{3}$ School of Physics, Korea Institute for Advanced Study, Seoul 02455, Korea
}

(Received 3 May 2019; accepted 27 January 2020; published 13 February 2020)

\begin{abstract}
Recently it has been noticed that the Fermi-LAT data of gamma-rays from some galactic pulsars and supernova remnants reveal spectral modulations that might be explained by the conversion of photons to ALPs (axionlike particles) induced by the conventional ALP coupling to photon in the presence of galactic magnetic fields. However the corresponding ALP mass and coupling are in a severe tension with the observational constraints from CAST, SN1987A, and other gamma-ray observations. Motivated by this, we examine an alternative possibility that those spectral modulations are explained by other type of ALP coupling involving both the ordinary photon and a massless dark photon, when nonzero background dark photon gauge fields are assumed. We find that our scheme results in oscillations among the photon, ALP, and dark photon, which can explain the gamma-ray spectral modulations of galactic pulsars or supernova remnants, while satisfying the known observational constraints.
\end{abstract}

DOI: 10.1103/PhysRevD.101.043007

\section{INTRODUCTION}

Light axionlike particles (ALPs) and hidden $U(1)$ gauge bosons have been widely discussed as well-motivated candidates for physics beyond the Standard Model of particle physics [1]. An appealing feature of those particles is that their lightness is protected from unknown UV physics by symmetry. They are also a good candidate for dark matter, and can result in a variety of other astrophysical and cosmological consequences. One of such phenomena associated with ALP is the photon to ALP conversions [2] in the presence of background magnetic fields, which may cause spectral modulations of x-rays [3-6] or gamma-rays [7-11] from pointlike sources, spectral distortion of CMB [12-15], change of the cosmic opacity of intergalactic medium [16-25], etc.

Recently it has been noticed that the Fermi-LAT data of high energy gamma-rays from some galactic pulsars [7] and supernova remnants [8] reveal an intriguing spectral

\footnotetext{
*kchoi@ibs.re.kr

†simflight@kaist.ac.kr

*sbravos@kaist.ac.kr

${ }^{\S}$ SeokhoonYun@kias.re.kr
}

Published by the American Physical Society under the terms of the Creative Commons Attribution 4.0 International license. Further distribution of this work must maintain attribution to the author(s) and the published article's title, journal citation, and DOI. Funded by SCOAP ${ }^{3}$. modulation (or irregularity) which might be explained by the photon to ALP conversions caused by the coupling

$$
\frac{1}{4} g_{a \gamma \gamma} a F_{\mu \nu} \tilde{F}^{\mu \nu}=-g_{a \gamma \gamma} a \vec{E} \cdot \vec{B}
$$

in the presence of galactic magnetic fields, where $F_{\mu \nu}=$ $\partial_{\mu} A_{\nu}-\partial_{\nu} A_{\mu}$ is the gauge field strength of the ordinary $U(1)_{\text {em }}$ gauge field $A_{\mu}$, and $\tilde{F}^{\mu \nu}=\frac{1}{2} \epsilon^{\mu \nu \rho \sigma} F_{\rho \sigma}$ is its dual. However the ALP mass $m_{a} \simeq$ few $\times 10^{-9} \mathrm{eV}$ and the coupling $g_{\text {ary }} \simeq$ few $\times 10^{-10} \mathrm{GeV}^{-1}$, which are required to explain those modulations, are in a severe tension with the CAST bound $g_{\text {ary }}<6.6 \times 10^{-11} \mathrm{GeV}^{-1}$ [26], as well as with the absence of gamma-ray bursts associated with SN1987A [27] and the Fermi-LAT data of gamma-rays from the galactic nucleus NGC 1275 in the center of the Perseus cluster $[9,10]$.

Motivated by this observation, in this paper we examine an alternative scenario to explain those gamma-ray spectral modulations by means of the photon-ALP-dark photon oscillations caused by the ALP coupling [28,29]

$$
\frac{1}{2} g_{a \gamma \gamma^{\prime}} a X_{\mu \nu} \tilde{F}^{\mu \nu}=-g_{a \gamma \gamma^{\prime}} a\left(\vec{E} \cdot \vec{B}_{X}+\vec{B} \cdot \vec{E}_{X}\right)
$$

in the presence of background $\left\langle B_{X}\right\rangle$ and/or $\left\langle E_{X}\right\rangle$, where $X_{\mu \nu}=\partial_{\mu} X_{\nu}-\partial_{\nu} X_{\mu}=\left(\vec{E}_{X}, \vec{B}_{X}\right)$ is the gauge field strength of a massless dark photon gauge field $X_{\mu}$. Like the ordinary ALP coupling (1), the above ALP coupling involving both 
the ordinary photon and dark photon generically exists once a dark photon field is introduced in the theory. As we will see, this coupling induces the conversion of gammarays to ALPs or dark photons, which can successfully explain the gamma-ray spectral modulations noticed in [7] and [8] for certain values of the ALP parameters and background dark photon gauge field in the range

$$
\begin{aligned}
m_{a} & \simeq(3-7) \times 10^{-9} \mathrm{eV}, \\
g_{\text {ary }} & \simeq 10^{-11}-10^{-10} \mathrm{GeV}^{-1}, \\
\left\langle B_{X}\right\rangle & \simeq 1-10 \mu \mathrm{G} .
\end{aligned}
$$

As we will show later, contrary to the scheme based on the ordinary ALP coupling $g_{a \gamma \gamma}$, our scheme can be compatible with all the available constraints on the scheme.

A key ingredient of our scenario is the existence of a background dark photon gauge fields $\left\langle B_{X}\right\rangle$ and/or $\left\langle E_{X}\right\rangle$ with a strength comparable to (or bigger than) the galactic magnetic fields $\sim 1 \mu \mathrm{G}$ and a coherent length longer than the typical galactic size $\sim 1 \mathrm{kpc}$. If they were generated in the early universe, such background dark photon gauge fields may result in a dangerous distortion of $\mathrm{CMB}$ due to the resonant conversion of CMB photons to axions, which would occur when the effective photon mass $m_{\gamma}(t)$ in the early universe crosses the ALP mass $m_{a}$ [13-15]. To avoid this problem, we need to generate the background dark photon gauge fields at a late time with $m_{\gamma}(t)<m_{a}$. In this paper, we will introduce an explicit model [30-38] for such a late generation of the background dark photon gauge fields, involving an additional ultralight ALP $\phi$ with $m_{\phi} \lesssim 10^{-27} \mathrm{eV}$, whose late coherent oscillations cause a tachyonic instability of the dark photon gauge fields and amplify the vacuum fluctuations to the desired background fields $\left\langle B_{X}\right\rangle \sim\left\langle E_{X}\right\rangle \gtrsim 1 \mu \mathrm{G}$.

This paper is organized as follows. In Sec. II, we discuss the photon-ALP-dark photon oscillations induced by the coupling (2) in the presence of background $\left\langle B_{X}\right\rangle$ and/or $\left\langle E_{X}\right\rangle$. In Sec. III, we examine if the gamma-ray spectral modulations noticed in [7] and [8] can be explained by the conversion of gamma-rays to ALPs or dark photons, which results from the photon-ALP-dark photon oscillations. In Sec. IV, we discuss the observational constraints on the ALP coupling (2) and examine if our scenario can be compatible with those constraints. Obviously the ALP coupling $g_{a y \gamma^{\prime}}$ is free from the bound from helioscope axion search experiments such as CAST. Yet it should satisfy the bound from stellar evolution [39], as well as another bound from the nonobservation of gamma-ray bursts associated with SN1987A [27]. We also need to generate the background dark photon gauge fields at a late time with $m_{\gamma}(t)<m_{a}$ to avoid a dangerous distortion of CMB [13-15]. In the later part of Sec. IV, we present an explicit model for such a late generation of the background dark photon gauge fields. Section V is the conclusion.

\section{PHOTON-ALP-DARK PHOTON OSCILLATIONS}

Our scheme is based on a model involving a light ALP and also a massless hidden $U(1)_{X}$ gauge boson $X_{\mu}$, which is dubbed the dark photon in this paper [1]. We assume that there is no light $U(1)_{X}$-charged matter fields. Here we are interested in the photon-ALP-dark photon oscillations induced by the ALP coupling $[28,29]$

$$
\frac{1}{2} g_{a \gamma \gamma^{\prime}} a X_{\mu \nu} \tilde{F}^{\mu \nu}=-g_{a \gamma \gamma^{\prime}} a\left(\vec{E} \cdot \vec{B}_{X}+\vec{B} \cdot \vec{E}_{X}\right)
$$

in the presence of nonzero background dark photon gauge fields, $\left\langle E_{X}\right\rangle$ and/or $\left\langle B_{X}\right\rangle$, as well as an ordinary background magnetic field $\langle B\rangle$. Since there is no $U(1)_{X}$-charged matter, $\left\langle E_{X}\right\rangle$ can be nonvanishing and then the equations of motion of $X_{\mu}$ suggest $\left\langle E_{X}\right\rangle \sim\left\langle B_{X}\right\rangle$ [34].

Generically there can be other ALP couplings such as $g_{a \gamma \gamma} a F \tilde{F}$ and $g_{a \gamma^{\prime} \gamma^{\prime}} a X \tilde{X}$. For simplicity, here we will assume that $g_{a \gamma \gamma^{\prime}}$ dominates over $g_{a \gamma \gamma}$ and $g_{a \gamma^{\prime} \gamma^{\prime}}$ as much as we need. Note that in many cases the ALP couplings to gauge fields are quantized as they are generated by the loops of fermions carrying quantized charges of the $U(1)_{\mathrm{PQ}}, U(1)_{\mathrm{em}}$, and $U(1)_{X}$, where $U(1)_{\mathrm{PQ}}$ denotes the (spontaneously broken) global $U(1)$ symmetry associated with the ALP field " $a$ ". In those cases, one can arrange the relevant PQ and gauge charges appropriately to get the desired pattern of ALP couplings such as $\left|g_{a \gamma \gamma^{\prime}}\right| \gtrsim\left|g_{a \gamma^{\prime} \gamma^{\prime}}\right| \gg\left|g_{a \gamma \gamma}\right|$. Alternatively, one may use the clockwork mechanism [40-43] to generate such pattern of ALP couplings as was done in [44-46] to get an axion-photon coupling much stronger than the axion-gluon coupling.

To examine the oscillations among the photon, dark photon and ALP, let us choose the temporal gauge $A_{0}=X_{0}=0$. Then the equations of motion describing the propagation of small field fluctuations of $\vec{A}, \vec{X}$, and $a$ in the background $\langle\vec{B}\rangle,\left\langle\vec{B}_{X}\right\rangle$, and $\left\langle\vec{E}_{X}\right\rangle$ are given by

$$
\begin{aligned}
\partial_{\mu} \partial^{\mu} \vec{A}= & g_{a \gamma \gamma^{\prime}}\left(\left\langle\vec{B}_{X}\right\rangle \partial_{t} a-\left\langle\vec{E}_{X}\right\rangle \times \nabla a\right), \\
\partial_{\mu} \partial^{\mu} \vec{X}= & g_{a \gamma \gamma^{\prime}}\langle\vec{B}\rangle \partial_{t} a, \\
\partial_{\mu} \partial^{\mu} a+m_{a}^{2} a= & -g_{a \gamma \gamma^{\prime}}\left(\langle\vec{B}\rangle \cdot \partial_{t} \vec{X}+\left\langle\vec{B}_{X}\right\rangle \cdot \partial_{t} \vec{A}\right. \\
& \left.-\left\langle\vec{E}_{X}\right\rangle \cdot \nabla \times \vec{A}\right) .
\end{aligned}
$$

From the above wave equations, we note that the following transverse background fields induce oscillations among the photon, dark photon, and ALP:

$$
\begin{aligned}
\vec{B}_{T} & =\langle\vec{B}\rangle-\hat{k}(\hat{k} \cdot\langle\vec{B}\rangle), \\
\vec{B}_{\mathrm{XT}} & =\left\langle\vec{B}_{X}\right\rangle-\hat{k}\left(\hat{k} \cdot\left\langle\vec{B}_{X}\right\rangle\right)-\hat{k} \times\left\langle\vec{E}_{X}\right\rangle,
\end{aligned}
$$


where $\vec{k}$ is the momentum of the propagating particles and $\hat{k}=\vec{k} /|\vec{k}|$. In the relativistic limit, the corresponding propagation equation is well approximated by [2]

$$
\left[w+i \partial_{z}-\mathcal{M}\right]\left(\begin{array}{c}
A_{\|} \\
X_{\|} \\
a
\end{array}\right)=0,
$$

where $\omega=|\vec{k}|$ and $z$ is the spacial coordinate along the direction $\hat{k}$. Here $A_{\|}$and $X_{\|}$denote the polarization of $\vec{A}$ and $\vec{X}$ in the direction of $\vec{B}_{\mathrm{XT}}$ and $\vec{B}_{T}$, respectively:

$$
A_{\|}=\frac{\vec{B}_{\mathrm{XT}} \cdot \vec{A}}{B_{\mathrm{XT}}}, \quad X_{\|}=\frac{\vec{B}_{T} \cdot \vec{X}}{B_{T}},
$$

and

$$
\mathcal{M}=\left(\begin{array}{ccc}
\frac{\omega_{\mathrm{pl}}^{2}}{2 \omega} & 0 & \frac{g_{a \gamma \gamma^{\prime}} B_{\mathrm{XT}}}{2} \\
0 & 0 & \frac{g_{a \gamma \gamma^{\prime}} B_{T}}{2} \\
\frac{g_{a \gamma \gamma^{\prime}} B_{\mathrm{XT}}}{2} & \frac{g_{a \gamma \gamma^{\prime}} B_{T}}{2} & \frac{m_{a}^{2}}{2 \omega}
\end{array}\right),
$$

where $\omega_{\mathrm{pl}}^{2}=4 \pi \alpha n_{e} / m_{e}$ is the plasma frequency in a medium with the electron number density $n_{e}$.

Here we are mostly interested in the conversion of initial unpolarized photon to ALP or dark photon in the limit when the plasma frequency is negligible:

$$
\omega_{\mathrm{pl}}^{2} \ll \min \left(m_{a}^{2}, g_{a \gamma \gamma^{\prime}} \omega \sqrt{B_{T}^{2}+B_{\mathrm{XT}}^{2}}\right),
$$

which turns out to be the case for our scenario to explain the gamma-ray spectral modulations noticed in [7] and [8]. To get some insights on the photon-ALP-dark photon oscillations, let us consider a simple situation that all background fields have a coherent length which is large enough to treat them as constants over the photon propagation distance $d$. It is then straightforward to find that the conversion probabilities in such case are given by

$$
\begin{aligned}
P_{\gamma \rightarrow a}= & P_{a \rightarrow \gamma}=\left(\frac{B_{\mathrm{XT}}^{2}}{B_{\mathrm{XT}}^{2}+B_{T}^{2}}\right)\left(\frac{\omega^{2}}{\omega^{2}+\omega_{c}^{2}}\right) \sin ^{2} \frac{\Delta_{\mathrm{osc}} d}{2}, \\
P_{\gamma \rightarrow \gamma^{\prime}}= & P_{\gamma^{\prime} \rightarrow \gamma}=\frac{2 B_{\mathrm{XT}}^{2} B_{T}^{2}}{\left(B_{\mathrm{XT}}^{2}+B_{T}^{2}\right)^{2}}\left(1-\cos \frac{\Delta_{a} d}{2} \cos \frac{\Delta_{\mathrm{osc}} d}{2}\right. \\
& -\frac{\omega_{c}}{\sqrt{\omega^{2}+\omega_{c}^{2}}} \sin \frac{\Delta_{a} d}{2} \sin \frac{\Delta_{\mathrm{osc}} d}{2} \\
& \left.-\frac{\omega^{2}}{2\left(\omega^{2}+\omega_{c}^{2}\right)} \sin ^{2} \frac{\Delta_{\mathrm{osc}} d}{2}\right),
\end{aligned}
$$

where $d$ is the propagation distance, and

$$
\begin{aligned}
\omega_{c} & =\frac{m_{a}^{2}}{2 g_{a \gamma \gamma^{\prime}} B_{\mathrm{eff}}}, \quad \Delta_{a}=g_{a \gamma \gamma^{\prime}} B_{\mathrm{eff}} \frac{\omega_{c}}{\omega}, \\
\Delta_{\mathrm{osc}} & =g_{a \gamma \gamma^{\prime}} B_{\mathrm{eff}} \sqrt{1+\left(\frac{\omega_{c}}{\omega}\right)^{2}}
\end{aligned}
$$

for

$$
B_{\text {eff }} \equiv \sqrt{B_{\mathrm{XT}}^{2}+B_{T}^{2}}
$$

From the above results, we find the following photon survival probability for the photon-ALP-dark photon oscillations induced by the ALP coupling $g_{a \gamma \gamma}$ :

$$
\begin{aligned}
\left(P_{\gamma \rightarrow \gamma}\right)_{g_{a \gamma \gamma^{\prime}}}= & \frac{B_{\mathrm{XT}}^{4}+B_{T}^{4}}{\left(B_{\mathrm{XT}}^{2}+B_{T}^{2}\right)^{2}} \\
& -\left(\frac{B_{\mathrm{XT}}^{2}}{B_{\mathrm{XT}}^{2}+B_{T}^{2}}\right)^{2}\left(\frac{\omega^{2}}{\omega^{2}+\omega_{c}^{2}}\right) \sin ^{2} \frac{\Delta_{\mathrm{osc}} d}{2} \\
& +2\left(\frac{B_{\mathrm{XT}} B_{T}}{B_{\mathrm{XT}}^{2}+B_{T}^{2}}\right)^{2}\left(\cos \frac{\Delta_{a} d}{2} \cos \frac{\Delta_{\mathrm{osc}} d}{2} .\right. \\
& \left.+\frac{\omega_{c}}{\sqrt{\omega^{2}+\omega_{c}^{2}}} \sin \frac{\Delta_{a} d}{2} \sin \frac{\Delta_{\mathrm{osc}} d}{2}\right) .
\end{aligned}
$$

For later discussion of the possibility that the ALP coupling $g_{a r \gamma^{\prime}}$ explains the gamma ray modulations noticed in [7] and [8], let us compare the photon depletion caused by $g_{\text {arr' }}$ in background $U(1)_{X}$ gauge fields with the photon depletion by $g_{a \gamma y}$ in ordinary magnetic fields. As is well known [2], the photon survival probability for the photonALP oscillations caused by $g_{a \gamma \gamma}$ in ordinary magnetic fields is given by

$$
\left(P_{\gamma \rightarrow \gamma}\right)_{g_{a \gamma \gamma}}=1-\left(\frac{\omega^{2}}{\omega^{2}+\tilde{\omega}_{c}^{2}}\right) \sin ^{2} \frac{\tilde{\Delta}_{\mathrm{osc}} d}{2},
$$

where

$\tilde{\omega}_{c}=\frac{m_{a}^{2}}{2 g_{a \gamma \gamma} B_{T}}, \quad \tilde{\Delta}_{\mathrm{osc}}=g_{a \gamma \gamma} B_{T} \sqrt{1+\left(\frac{\tilde{\omega}_{c}}{\omega}\right)^{2}}$.

There are two key spectral features of the above photon survival probability. The first is the step-down of $P_{\gamma \rightarrow \gamma}$ at $\omega \sim \tilde{\omega}_{c}$, which represents the transition from the small mixing limit $\omega \ll \tilde{\omega}_{c}$ to the large mixing limit $\omega \gg \tilde{\omega}_{c}$. The size of step-down is given by

$$
\begin{aligned}
\Delta P & \equiv P_{\gamma \rightarrow \gamma}\left(\omega \ll \tilde{\omega}_{c}\right)-P_{\gamma \rightarrow \gamma}\left(\omega \gg \tilde{\omega}_{c}\right) \\
& =\sin ^{2}\left(\frac{g_{a \gamma \gamma} B_{T} d}{2}\right),
\end{aligned}
$$


which can be significant when $g_{a \gamma \gamma} B_{T} d \gtrsim \mathcal{O}(1)$. The second feature of $\left(P_{\gamma \rightarrow \gamma}\right)_{g_{a \gamma \gamma}}$ is the oscillatory behavior due to $\sin ^{2}\left(\tilde{\Delta}_{\text {osc }} d / 2\right)$, which becomes appreciable right before the step-down. The spectral modulations (irregularities) noticed in [7] and [8] could result from the combination of these two effects on the gamma-ray intensity around $\omega=\mathcal{O}(1) \mathrm{GeV}$.

The photon survival probability $\left(P_{\gamma \rightarrow \gamma}\right)_{g_{a \gamma \gamma^{\prime}}}$ of (14) appears to be significantly more complicated than $\left(P_{\gamma \rightarrow \gamma}\right)_{g_{a \gamma \gamma}}$ of (15). Yet, as long as $B_{\mathrm{XT}}$ is not negligible compared to $B_{T}$, it reveals a similar step-down behavior at $\omega \sim \omega_{c}$, as well as a similar oscillatory feature. To see this, let us first consider the case $B_{\mathrm{XT}} \gg B_{T}$. In such case, the dark photon is decoupled and the resulting photon-ALP oscillation yields

$\left(P_{\gamma \rightarrow \gamma}\right)_{g_{a \gamma \gamma^{\prime}}} \simeq 1-\left(\frac{\omega^{2}}{\omega^{2}+\omega_{c}^{2}}\right) \sin ^{2}\left(\frac{g_{a \gamma \gamma^{\prime}} B_{\mathrm{XT}} d}{2} \sqrt{1+\frac{\omega_{c}^{2}}{\omega^{2}}}\right)$

for $B_{T} \ll B_{\mathrm{XT}}$. Obviously the above $\left(P_{\gamma \rightarrow \gamma}\right)_{g_{a \gamma \gamma^{\prime}}}$ mimics $\left(P_{\gamma \rightarrow \gamma}\right)_{g_{a \gamma \gamma}}$ with the simple correspondence

$$
g_{a \gamma \gamma^{\prime}} B_{\mathrm{XT}} \rightarrow g_{a \gamma \gamma} B_{T}
$$

Even when $B_{\mathrm{XT}} \lesssim B_{T}$, if it is non-negligible, there can be a significant step-down of $\left(P_{\gamma \rightarrow \gamma}\right)_{g_{a \gamma \gamma^{\prime}}}$ at $\omega \sim \omega_{c}$. From (14), one finds

$$
\left(P_{\gamma \rightarrow \gamma}\right)_{g_{a \gamma \gamma^{\prime}}}\left(\omega \ll \omega_{c}\right) \simeq 1-\frac{4 B_{\mathrm{XT}}^{2} B_{T}^{2}}{\left(B_{\mathrm{XT}}^{2}+B_{T}^{2}\right)^{2}} \sin ^{2}\left(\frac{g_{a \gamma \gamma^{\prime}} B_{\mathrm{eff}} d}{8} \frac{\omega}{\omega_{c}}\right)
$$

and

$$
\begin{aligned}
\left(P_{\gamma \rightarrow \gamma}\right)_{g_{a \gamma \gamma^{\prime}}}\left(\omega \gg \omega_{c}\right) \simeq & 1-\frac{B_{\mathrm{XT}}^{4}}{\left(B_{\mathrm{XT}}^{2}+B_{T}^{2}\right)^{2}} \sin ^{2}\left(\frac{g_{a \gamma \gamma^{\prime}} B_{\mathrm{eff}} d}{2}\right) \\
& -\frac{2 B_{\mathrm{XT}}^{2} B_{T}^{2}}{\left(B_{\mathrm{XT}}^{2}+B_{T}^{2}\right)^{2}}\left(1-\cos \left(\frac{g_{a \gamma \gamma^{\prime}} B_{\mathrm{eff}} d}{2}\right)\right. \\
& \left.\times \cos \left(\frac{g_{a \gamma \gamma^{\prime}} B_{\mathrm{eff}} d}{2} \frac{\omega_{c}}{\omega}\right)\right) .
\end{aligned}
$$

When $g_{a \gamma \gamma^{\prime}} B_{\text {eff }} d=\mathcal{O}(1)$, which is the case relevant for us, this results in the following step-down of the photon survival probability:

$$
\begin{aligned}
\Delta P= & P_{\gamma \rightarrow \gamma}\left(\omega \ll \omega_{c}\right)-P_{\gamma \rightarrow \gamma}\left(\omega \gg \omega_{c}\right) \\
\simeq & \frac{4 B_{\mathrm{XT}}^{2} B_{T}^{2}}{\left(B_{\mathrm{XT}}^{2}+B_{T}^{2}\right)^{2}} \sin ^{2}\left(\frac{g_{a \gamma \gamma^{\prime}} B_{\mathrm{eff}} d}{4}\right) \\
& +\frac{B_{\mathrm{XT}}^{4}}{\left(B_{\mathrm{XT}}^{2}+B_{T}^{2}\right)^{2}} \sin ^{2}\left(\frac{g_{a \gamma \gamma^{\prime}} B_{\mathrm{eff}} d}{2}\right) .
\end{aligned}
$$

This step-down of the photon survival probability can be sizable enough to yield an observational consequence if $B_{\mathrm{XT}}$ is comparable to $B_{T}$.

The discussions above indicate that the results of [7] and [8], which are based on the ALP coupling $g_{a \gamma \gamma}$, can be reproduced also by $g_{a \gamma \gamma^{\prime}}$ for appropriate range of ALP parameters if the background dark photon gauge fields $E_{X}$ and/or $B_{X}$ are comparable to or even larger than the galactic magnetic fields $B \sim 1 \mu \mathrm{G}$. In the next section, we examine this possibility in more detail for specific gamma-ray sources, the galactic pulsar J2021 + 3651, and the supernova remnant IC443.

\section{GAMMA-RAY SPECTRAL MODULATIONS}

In [7] and [8], Fermi-LAT data of gamma-rays from some galactic pulsars with a distance $d \simeq 5-10 \mathrm{kpc}$ and supernova remnants with $d \simeq 1-5 \mathrm{kpc}$ were examined to see if there is any irregularity in the spectrum. It was then noticed that the data indicates spectral modulations at $\omega \sim 1 \mathrm{GeV}$, which might be explained by the photon-ALP oscillations induced by an ALP coupling $g_{\text {ary }} \sim$ few $\times 10^{-10} \mathrm{GeV}^{-1}$ in the presence of galactic magnetic fields $B \sim 1 \mu \mathrm{G}$ for ALP mass $m_{a} \sim$ few $\times 10^{-9} \mathrm{eV}$. However these ALP parameters are in conflict with the bound from CAST [26], as well as with the nonobservation of gamma-ray bursts associated with SN1987A [27]. This motivates us an alternative scenario that those spectral modulations are explained by the photon-ALP-dark photon oscillations induced by $g_{\text {ary' }}$, which were discussed in the previous section.

In the previous section, the conversion probabilities for the photon-ALP-dark photon oscillations have been derived when the background gauge fields are assumed to be constant. In the following, we take a more elaborate approach taking into account the spacial variation of galactic magnetic fields along the line of sight toward the gamma-ray sources, and solve the propagation equation (7) numerically. Specifically, we adopt the latest model for galactic profile of the magnetic field, i.e., the Jansson and Farrar model [47], which was adopted also in [7] and [8]. As for the background dark photon gauge field component $B_{\mathrm{XT}}$, we simply assume that it is homogeneous enough ${ }^{1}$ to be treated as constant over the galactic distance scales of $\mathcal{O}(1) \mathrm{kpc}$.

Let us now examine in more detail if our photon-ALPdark photon oscillation scenario can explain the observed gamma-ray spectral modulations in the spectra of PSR $\mathrm{J} 2021+3651$ and IC443. For this, we perform a simplified $\chi^{2}$ analysis over the parameter space including the ALP mass $m_{a}$, ALP coupling $g_{a \gamma \gamma^{\prime}}$, and the fitting parameters of the intrinsic spectrum. Here the simplified analysis means

\footnotetext{
${ }^{1}$ Indeed our mechanism to generate the background dark photon gauge fields, which will be presented in the later part of the next section, yields a coherent length $\lambda \gg \mathrm{kpc}$ of the produced $B_{\mathrm{XT}}$, justifying this assumption.
} 
that we ignore the dispersion matrix effect which would take into account of systematic errors on the reconstruction of the true energy. Based on the Fermi data instruction on the energy dispersion, ${ }^{2}$ the instrument response effects are expected to give a few percent level of change in the spectral parameters for the energy range in our case. As the main goal of this work is to propose a new theoretical idea, more refined $\chi^{2}$ analysis taking into account of the full systematic uncertainties is beyond the scope of this work. Yet our simplified $\chi^{2}$ analysis is expected to be good enough to identify the parameter region which can explain the gamma-ray spectral modulations noticed in [7] and [8].

Figure 1 shows the result of our $\chi^{2}$ analysis for PSR $\mathrm{J} 2021+3651$ in our scenario. To be specific, here we assume $B_{\mathrm{XT}}=6.5 \mu \mathrm{G}$ (for the generation of such background dark photon gauge field, see Sec. IV D). We take the data from [7] and use the same setup as [7] for the galactic magnetic fields (Jansson and Farrar [47]) and the intrinsic spectrum (PLSuperExpCutoff). We also adopt the relative systematic uncertainty of $2.4 \%$ for the consistency with [7]. Then the best fit parameters are found to be

$$
\begin{aligned}
m_{a} & \simeq 4.0 \mathrm{neV}, \\
g_{\text {ary' }} & \simeq 4.3 \times 10^{-11} \mathrm{GeV}^{-1}
\end{aligned}
$$

for $B_{\mathrm{XT}}=6.5 \mu \mathrm{G}$.

In Fig. 2, we do the same $\chi^{2}$ analysis for IC443. We again assume $B_{\mathrm{XT}}=6.5 \mu \mathrm{G}$ and take the data from [8]. As for galactic magnetic fields, we use the Jansson and Farrar model [47]. However the intrinsic spectrum is modeled by LogParabola function ${ }^{3}$ and the relative systematic uncertainty is set by $3 \%$ for the consistency with [8]. We then find the best fit parameters for IC443 are given by

$$
\begin{aligned}
m_{a} & \simeq 6.0 \mathrm{neV}, \\
g_{a \gamma \gamma^{\prime}} & \simeq 5.5 \times 10^{-11} \mathrm{GeV}^{-1}
\end{aligned}
$$

for $B_{\mathrm{XT}}=6.5 \mu \mathrm{G}$.

Our results clearly show that the spectral modulations of galactic pulsars and supernova remnants noticed in [7] and [8] can be explained by the photon-ALP-dark photon oscillations caused by the ALP coupling $g_{a \gamma \gamma^{\prime}}$, with a significance similar to that of the explanation based on the ordinary ALP coupling $g_{a \gamma \gamma}$. As will be examined in detail in the next section, unlike the explanation based on $g_{a \gamma \gamma}$, our scheme can be compatible with the known observational constraints. It is remarkable that although the distance $d \simeq 1.5 \mathrm{kpc}$ from IC443 is quite shorter than the distance $d \simeq 10 \mathrm{kpc}$ from $\mathrm{J} 2021+3651$, the best fit point of $\left(m_{a}, g_{a \gamma \gamma^{\prime}}\right)$ to explain the spectral irregularity of IC443 is close to the best fit point of J2021 + 3651 for

\footnotetext{
$2 /$ analysis/documentation/Pass8_edisp_usage.html.

3 /analysis/scitools/source_models.html.
}
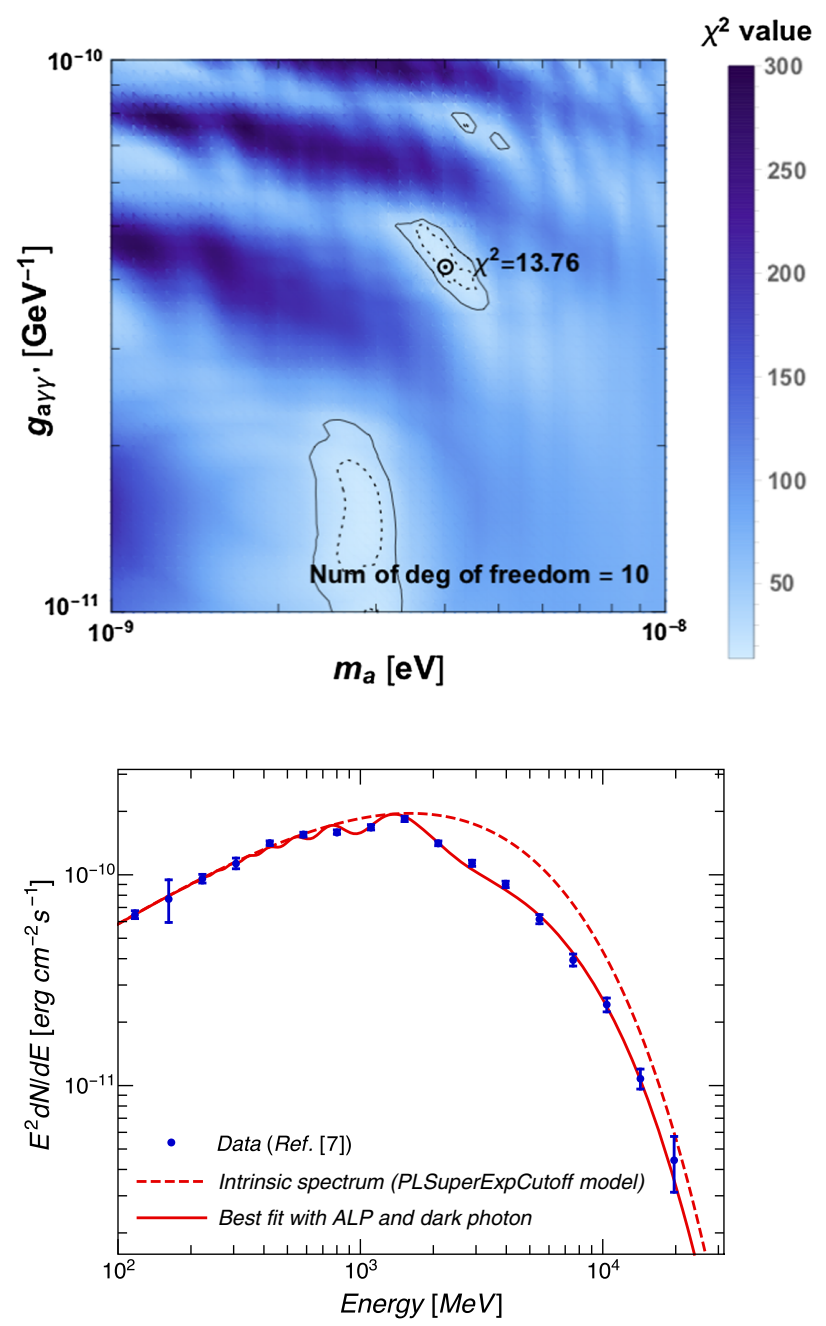

FIG. 1. (Top) $\chi^{2}$ values for PSR J2021 +3651 with varying $m_{a}$, $g_{a y \gamma^{\prime}}$ and the intrinsic spectrum parameters. Here the background dark photon gauge field is fixed as $B_{\mathrm{XT}}=6.5 \mu \mathrm{G}$ for simplicity. The solid (dotted) line represents the 1- $\sigma(2-\sigma)$ contour and $\odot$ denotes the best fit point for the data taken from [7]. (Bottom) Best fit spectrum (red solid line) for $B_{\mathrm{XT}}=6.5 \mu \mathrm{G}$, $\log _{10}\left(m_{a} / \mathrm{eV}\right)=-8.396$, and $\log _{10}\left(g_{a \gamma \gamma^{\prime}} / \mathrm{GeV}^{-1}\right)=-10.37$, which correspond to $\odot$ in the top panel. Blue dots with error bars denote the data taken from [7]. For the intrinsic spectrum (red dashed line), we use the PLSuperExpCutoff model as [7].

$B_{\mathrm{XT}}=6.5 \mu \mathrm{G}$. Therefore we expect that there exists a region of $\left(m_{a}, g_{a \gamma \gamma^{\prime}}, B_{\mathrm{XT}}\right)$ which can explain the both spectral modulations with the same parameter values and large enough significance. Finding such parameter values requires an extended $\chi^{2}$ analysis with varying $B_{\mathrm{XT}}$, which is beyond the scope of this paper.

\section{OBSERVATIONAL CONSTRAINTS AND A LATE GENERATION OF THE BACKGROUND DARK PHOTON GAUGE FIELDS}

In this section, we examine if our photon-ALP-dark photon oscillation scenario discussed in the previous 

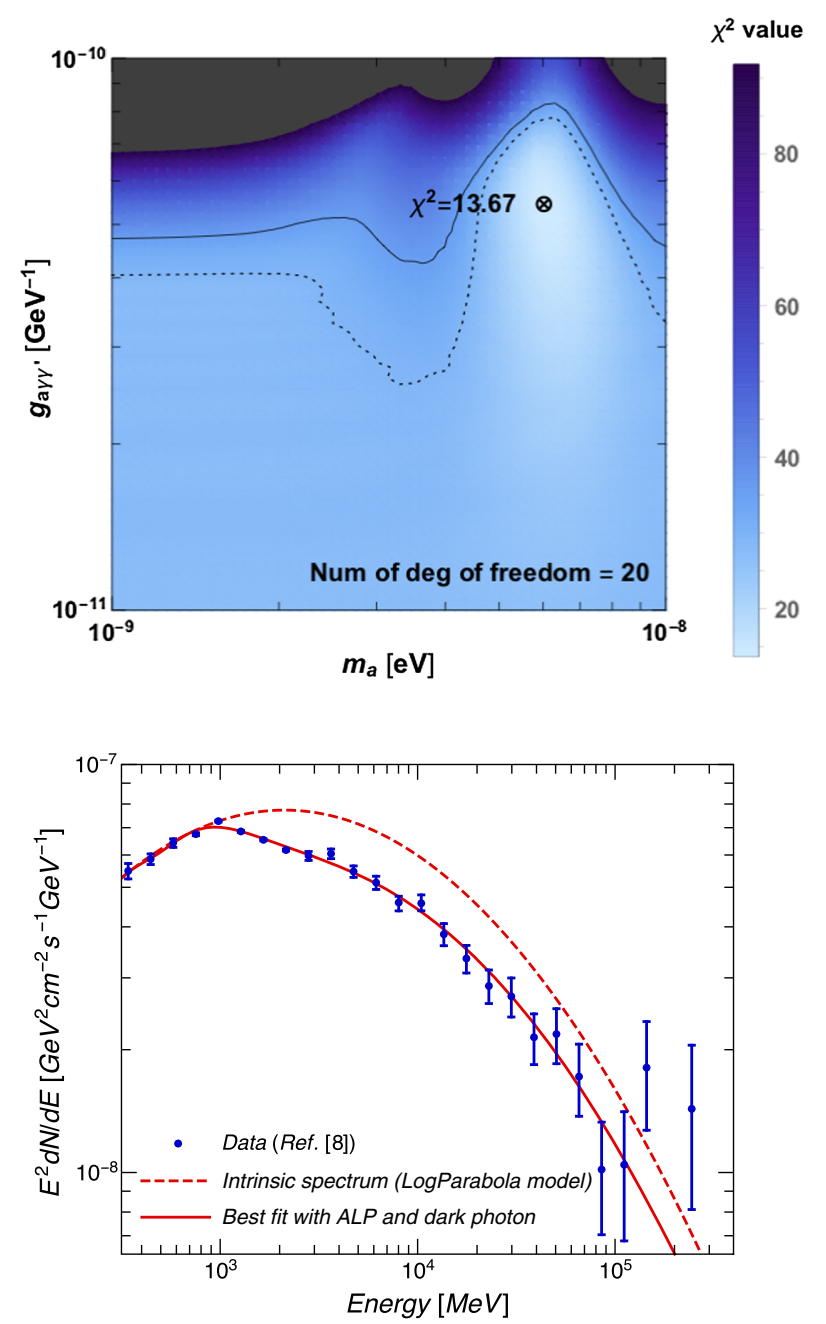

FIG. 2. (Top) $\chi^{2}$ values for IC443 with varying $m_{a}, g_{a \gamma \gamma^{\prime}}$ and the intrinsic spectrum parameters. Again the background dark photon gauge field is fixed as $B_{\mathrm{XT}}=6.5 \mu \mathrm{G}$, the solid (dotted) line represents the $1-\sigma(2-\sigma)$ contour, and $\otimes$ denotes the best fit point for the data taken from [8]. Dark gray region is cut out because of large $\chi^{2}$ which worsens the contrast in the figure. (Bottom) Best fit spectrum (red solid line) for $B_{\mathrm{XT}}=6.5 \mu \mathrm{G}, \log _{10}\left(m_{a} / \mathrm{eV}\right)=$ -8.22 , and $\log _{10}\left(g_{\text {ary }} / \mathrm{GeV}^{-1}\right)=-10.26$, which corresponds to $\otimes$ in the top panel. Blue dots with error bars denote the data taken from [8] and the LogParabola model is used for the intrinsic spectrum (red dashed line) as in [8].

section can be compatible with the observational constraints available at present. Note that the major problem of the previous explanation based on the ALP coupling $g_{\text {ayr }}$, which was suggested in [7] and [8], is the conflict with the bound on $g_{a \gamma \gamma}$ from CAST [26] and also the nonobservation of gamma-ray bursts from SN1987A [27]. ${ }^{4}$

\footnotetext{
${ }^{4}$ According to the recent analysis [10], the explanation by $g_{a \gamma \gamma}$ is disfavored also by the gamma-ray spectra of NGC 1275 , although it is within the allowed region of the previous analysis [9].
}

We first note that our ALP coupling $g_{a \gamma \gamma^{\prime}}$ does not cause a conversion of ALP to photon in background magnetic field, and therefore is not constrained by helioscopic axion search experiments such as CAST. It is yet constrained by a variety of astrophysical and/or cosmological observations, including the stellar evolution, the absence of gamma-ray bursts from SN1987A, and a distortion of CMB. In the following, we examine those constraints on $g_{a y \gamma^{\prime}}$ to see if the ALP parameters and the background dark photon gauge fields which would explain the gamma-ray modulations noticed in [7] and [8] can pass the observational constraints. In the later part of this section, we introduce an explicit model to generate the background dark photon gauge field $\left\langle B_{X}\right\rangle$ and/ or $\left\langle E_{X}\right\rangle$, which is a key ingredient of our scheme.

\section{A. Stellar evolutions}

Let us start with the constraint from stellar evolution. If light hidden sector particles are produced and successfully emitted from the core of star, such additional energy loss mechanism can affect the properties of the stellar object such as the core mass, luminosity, and lifetime [39]. In an ionized plasma such as the core of star, the excitations of the electromagnetic field possess a different dispersion relation due to the coherent interaction with medium, and the photon obtains an effective mass, the plasmon mass $\omega_{\mathrm{pl}}=\sqrt{4 \pi \alpha n_{e} / m_{e}}$. In the presence of the ALP coupling $g_{\text {arr' }}$, the massive plasmons decay into ALP and dark photon through the coupling $g_{a r \gamma^{\prime}}$. The corresponding decay width is given by

$$
\Gamma_{\mathrm{pl}}=\frac{g_{a \gamma \gamma^{\prime}}^{2}}{96 \pi} \frac{\omega_{\mathrm{pl}}^{4}}{\omega}\left[\beta\left(1, \frac{m_{a}}{\omega_{\mathrm{pl}}}\right)\right]^{3 / 2}
$$

where $\beta(x, y)=x^{4}+y^{4}-2 x^{2} y^{2}$. This results in the following energy loss rate per volume:

$$
Q_{\mathrm{pl}}=\frac{2}{2 \pi^{2}} \int_{0}^{\infty} d k k^{2} \frac{\omega \Gamma_{\mathrm{pl}}}{e^{\omega / T_{c}}-1},
$$

where $T_{c}$ is the core temperature. Here only the contributions from the decays of transverse plasmon modes are considered as the longitudinal modes have a negligible number density.

The most stringent constraint associated with the above plasmon decays comes from the observationally inferred core mass at the tip of red giant. Any extra cooling during the red-giant phase causes a delay of the helium ignition until the core mass at the helium flash reaches an adequate value, i.e. an increment of the core mass [48]. On the other hand, in view of the brightness at the tip of the red-giant branch in globular clusters, the core mass at the helium flash cannot exceed its standard value by more than 5\% [39]. Imposing this condition, the energy loss by plasmon decays is constrained as 


$$
\frac{Q_{\mathrm{pl}}}{\rho} \lesssim 10 \operatorname{erg~g}^{-1} \mathrm{~s}^{-1}
$$

which results in the following upper bound:

$g_{a \gamma \gamma^{\prime}} \lesssim 5 \times 10^{-10} \mathrm{GeV}^{-1}$ for $m_{a}, m_{X} \lesssim 10 \mathrm{keV}$,

where $\rho$ denotes the energy density of core, and $m_{X}$ denotes the dark photon mass which is assumed to be zero in our case.

\section{B. Gamma ray bursts from SN1987A}

In the presence of nonzero background dark photon fields, there can be additional constraints on $g_{a \gamma \gamma^{\prime}}$. One such example is the constraint from the gamma-ray bursts associated with SN1987A [27]. When the neutrino signals from SN1987A were observed, the Gamma-Ray Spectrometer on the Solar Maximum Mission satellite was operating to detect the $\gamma$-ray signals in the three energy bins, 4.1-6.4 MeV, 10-25 MeV, and 25-100 MeV, and found no appreciable gamma-ray excess [49]. This results in the following $95 \%$ confident upper bounds on the gamma ray fluence $\mathcal{F}_{\gamma}$ over the SN neutrino duration time $t_{\text {dur }} \simeq 10 \mathrm{sec}:$

$$
\mathcal{F}_{\gamma}<(0.9,0.4,0.6) \gamma \mathrm{cm}^{-2}
$$

for $(4.1-6.4,10-25,25-100) \mathrm{MeV}$.

In our case, the ALPs and dark photons are produced by the plasmon decays in SN1987A whose rate is given by (25). Accepting the core-collapse supernovae model with the $18 M_{\odot}$ progenitor star [50], the plasma frequency in the degenerate core of SN1987A is given by $\omega_{\mathrm{pl}}^{2} \simeq$ $4 \alpha \mu_{e}^{2} / 3 \pi$, where $\mu_{e}=5.15 \mathrm{keV}\left(Y_{e} \rho_{c}\right)^{1 / 3}$ is the electron chemical potential for the electron number per baryon $Y_{e} \simeq 0.2$ and the core density $\rho_{c} \simeq 3 \times 10^{14}$ (in the unit of $\mathrm{g} \mathrm{cm}^{-3}$ ). The average core temperature is $T_{c} \simeq 30 \mathrm{MeV}$ which is approximately constant within the core radius $r_{c} \simeq 10 \mathrm{~km}$ during the neutrino burst duration $t_{\text {dur }} \simeq 10 \mathrm{sec}$. The resulting ALP and dark photon flux per unit energy over $t_{\text {dur }}$ is given by

$$
\frac{d \Phi_{a, \gamma^{\prime}}}{d \omega}=\frac{1}{4 \pi d^{2}}\left(\frac{2 \omega}{\pi} \frac{\sqrt{4 \omega^{2}-\omega_{\mathrm{pl}}^{2}}}{e^{2 \omega / T_{c}}-1} \Gamma_{\mathrm{pl}}\right) \frac{4 \pi}{3} r_{c}^{3} t_{\mathrm{dur}}
$$

where $d \simeq 50 \mathrm{kpc}$ is the distance to SN1987A. For the ALP parameter range of our interest, the produced ALPs and dark photons escape freely from the $\mathrm{SN}$ core as their mean free path is considerably larger than $r_{c}$. We then find the following total ALP and dark photon fluxes in each energy bin: $\begin{aligned} \Phi_{a, \gamma^{\prime}}^{4.1-6.4 \mathrm{MeV}} & \simeq 6.2 \mathrm{~cm}^{-2} \times\left(g_{a \gamma \gamma^{\prime}} / 10^{-10} \mathrm{GeV}^{-1}\right)^{2}, \\ \Phi_{a, \gamma^{\prime}}^{10-25 \mathrm{MeV}} & \simeq 335 \mathrm{~cm}^{-2} \times\left(g_{a \gamma \gamma^{\prime}} / 10^{-10} \mathrm{GeV}^{-1}\right)^{2}, \\ \Phi_{a, \gamma^{\prime}}^{25-100 \mathrm{MeV}} & \simeq 361 \mathrm{~cm}^{-2} \times\left(g_{a \gamma \gamma^{\prime}} / 10^{-10} \mathrm{GeV}^{-1}\right)^{2} .\end{aligned}$

Part of the ALPs and dark photons emitted from SN1987A can be converted into photons via the oscillations fueled by the background $B_{\mathrm{XT}}$ and $B_{T}$, which would result in a gamma-ray burst whose differential flux is given by

$$
\begin{aligned}
\frac{d \Phi_{\gamma}}{d \omega} & =P_{a \rightarrow \gamma} \frac{d \Phi_{a}}{d \omega}+P_{\gamma^{\prime} \rightarrow \gamma} \frac{d \Phi_{\gamma^{\prime}}}{d \omega} \\
& =\left(P_{a \rightarrow \gamma}+P_{\gamma^{\prime} \rightarrow \gamma}\right) \frac{d \Phi_{a, \gamma^{\prime}}}{d \omega} .
\end{aligned}
$$

For the photon-ALP oscillation caused by $g_{a \gamma \gamma}$, the galactic magnetic fields $(\sim \mu \mathrm{G})$ are the dominant source of the conversion because the intergalactic magnetic fields $(<\mathrm{nG})$ are negligibly small. On the other hand, in our case of the photon-ALP-dark photon oscillations induced by $g_{a \gamma \gamma^{\prime}}$ for the ALP mass $m_{a}=\mathcal{O}\left(10^{-9}-10^{-10}\right) \mathrm{eV}$, the intergalactic space between SN1987A and our Milky way galaxy where $B_{\mathrm{XT}} \gg B_{T}$ mainly contributes to the conversions. Note that in our case $B_{\mathrm{XT}}$ is generated by a cosmological mechanism and therefore its value in intergalactic space is similar to the value inside the Milky way galaxy. For such intergalactic conversions, the dark photon is decoupled and the conversion probability is given by

$$
\begin{aligned}
P_{a \rightarrow \gamma}+P_{\gamma^{\prime} \rightarrow \gamma} & \simeq P_{a \rightarrow \gamma} \\
& \simeq \frac{\omega^{2}}{\omega^{2}+\omega_{c}^{2}} \sin ^{2}\left(\frac{g_{a \gamma \gamma^{\prime}} B_{\mathrm{XT}} \tilde{d}}{2}\right),
\end{aligned}
$$

where we assumed that the coherent length of the background $B_{\mathrm{XT}}$ is longer than the distance $\tilde{d} \simeq 50 \mathrm{kpc}$ between SN1987A and the earth. Indeed, if $B_{\mathrm{XT}}$ is generated by an additional ultralight ALP $\phi$ at a late time with $m_{\gamma}(t)<m_{a}=\mathcal{O}\left(10^{-9}-10^{-10}\right) \mathrm{eV}$, which will be presented at the end of this section, the resulting coherent length is much longer than $\tilde{d} \simeq 50 \mathrm{kpc}$.

Applying the upper bounds (29) to the photon flux obtained from (32), we can get an upper bound on $g_{a \gamma \gamma^{\prime}}$ for given values of $m_{a}$ and $B_{\mathrm{XT}}$. We depict the results in Fig. 3 for the background dark photon gauge field $B_{\mathrm{XT}}=6.5 \mu \mathrm{G}$, which was used in the previous section to explain the spectral irregularities of the galactic pulsar PSR J2021+ 3651 and the supernova remnant IC443. In Fig. 3, the red and blue colored regions are excluded by the stellar evolution constraint and the absence of gamma-ray bursts associated with SN1987A, respectively, and $\odot$ and $\otimes$ denote the ALP parameters which were chosen in the previous section as the benchmark points for PSR $\mathrm{J} 2021+3651$ and IC443, respectively. 


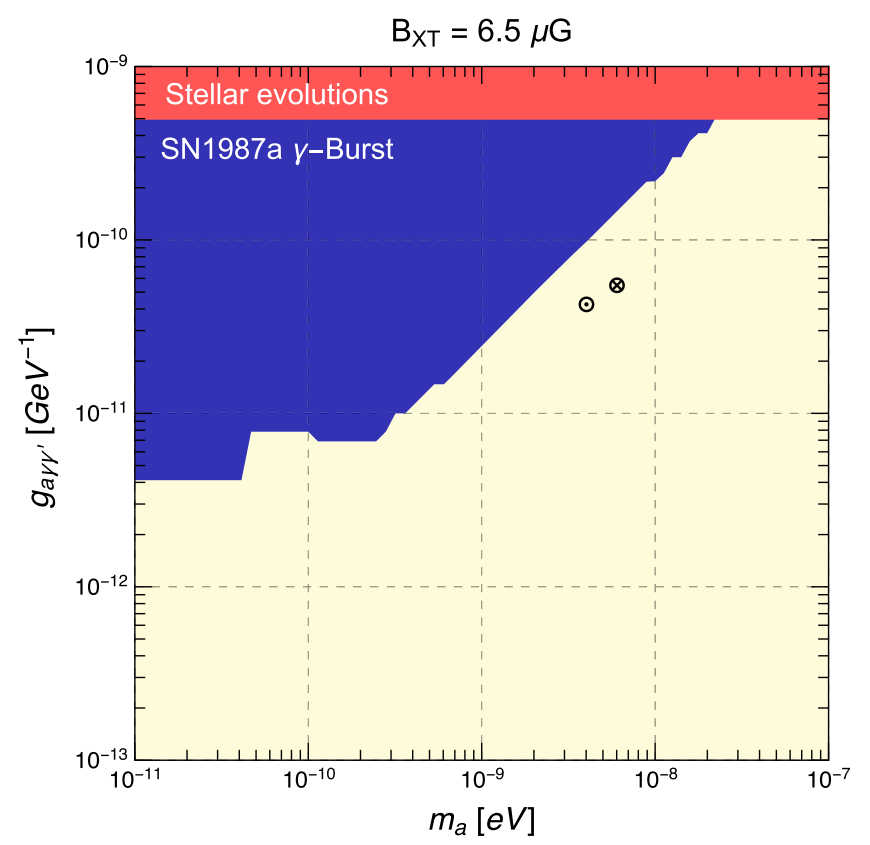

FIG. 3. Red and blue colored regions in the space of $\left(m_{a}, g_{a \gamma \gamma^{\prime}}\right)$ are excluded by stellar evolution and the absence of $\gamma$-ray bursts associated with SN1987A for the background dark photon gauge field $B_{\mathrm{XT}}=6.5 \mu \mathrm{G}$. The best fit values of $m_{a}$ and $g_{a \gamma \gamma^{\prime}}$ for the galactic pulsar PSR J2021 + 3651 and the supernova remnant IC443 given in (23) and (24) are marked with $\odot$ and $\otimes$, respectively.

\section{Other possible constraints}

In the previous subsections, we discussed the constraints on $g_{a r \gamma^{\prime}}$ from stellar evolution and the absence of $\gamma$-ray bursts associated with SN1987A. If the background dark photon gauge fields were generated early enough, e.g., in the early universe with an effective photon mass $m_{\gamma}(t)>m_{a}$, our ALP coupling $g_{a \gamma \gamma^{\prime}}$ is constrained also by the absence of a significant distortion of CMB.

It has been noticed that in the presence of the primordial cosmological background magnetic field $B_{0}$, the ALP coupling $g_{\text {ary }}$ can induce a resonant conversion of CMB photons to ALP when $m_{\gamma}(t)=m_{a}$, which would result in a distortion of the CMB spectrum, thereby providing a bound on the combination $g_{a \gamma \gamma} B_{0}$ [13-15]. The background dark photon field $B_{\mathrm{XT}} \gtrsim 1 \mu \mathrm{G}$ in our galaxy, which is required to explain the spectral irregularities of the galactic pulsars and supernova remnants noticed in [7] and [8], can not be amplified by the galactic dynamo mechanism [51] and therefore should have a cosmological origin. This implies that a similar size of background dark photon gauge fields exist over the entire universe. If such cosmological $B_{\mathrm{XT}}$ were generated in the early universe when $m_{\gamma}(t)>m_{a}=\mathcal{O}\left(10^{-9}\right) \mathrm{eV}$, so that $\mathrm{CMB}$ experiences a resonant conversion to ALP when $m_{\gamma}(t)=m_{a}$, the results of [13-15] can be straightforwardly applied to our case, yielding a bound $g_{a \gamma \gamma^{\prime}}\left\langle B_{\mathrm{XT}}\right\rangle<10^{-14} \mathrm{GeV}^{-1} \mu \mathrm{G}$ for an
ALP mass $m_{a}=\mathcal{O}\left(10^{-9}\right) \mathrm{eV}$, where $\left\langle B_{\mathrm{XT}}\right\rangle$ denotes the cosmic average of $B_{\mathrm{XT}}$. On the other hand, as was noticed in the previous section, we need $g_{a \gamma \gamma^{\prime}} B_{\mathrm{XT}}=$ $\mathcal{O}\left(10^{-10}\right) \mathrm{GeV}^{-1} \mu \mathrm{G}$ to explain the spectral irregularities of the galactic pulsars and supernova remnants, where now $B_{\mathrm{XT}}$ corresponds to the local dark photon field strength in our galaxy. As the local dark photon field strength is expected to be similar to its cosmic average, this implies that the background dark photon gauge fields should be generated at a late time when $m_{\gamma}(t)<m_{a}=\mathcal{O}\left(10^{-9}\right) \mathrm{eV}$, i.e., at the redshift

$$
z_{X}<z_{\text {res }} \simeq \text { few } \times 10^{3}
$$

where $z_{X}$ denotes the redshift factor at the time when the background dark photon gauge fields are produced, and $z_{\text {res }}$ is the redshift factor when $m_{\gamma}(t)=m_{a}=\mathcal{O}\left(10^{-9}\right) \mathrm{eV}$.

Even in such case avoiding dangerous resonant conversion, $\mathrm{CMB}$ distortion can occur through nonresonant conversion. The corresponding conversion probability can be easily estimated as

$$
P_{\gamma \rightarrow a}^{\text {non-res }} \sim \frac{g_{a \gamma \gamma^{\prime}}^{2} B_{\mathrm{XT}}^{2} \omega_{\mathrm{CMB}}^{2}}{m_{a}^{4}} \lesssim 10^{-8},
$$

where $\omega_{\mathrm{CMB}}$ denotes the $\mathrm{CMB}$ frequency and we used the best fit values of $\left(m_{a}, g_{a \gamma \gamma^{\prime}}, B_{\mathrm{XT}}\right)$ and $z_{X} \lesssim 10^{3}$ for the numerical estimate of the conversion probability. The above value of $P_{\gamma \rightarrow a}^{\text {non-res }}$ is well below the COBE-FIRAS sensitivity $\sim 10^{-4}$ [52], and therefore our scenario is safe from the spectral distortion of CMB.

As the background dark photon gauge fields were produced over the entire universe, they constitute a dark radiation whose energy density can be parametrized in the unit of the energy density of an additional relativistic neutrino species as follows:

$$
\Delta N_{\mathrm{eff}}(X) \simeq 0.42 \frac{\left\langle E_{X}^{2}\right\rangle+\left\langle B_{X}^{2}\right\rangle}{(1 \mu \mathrm{G})^{2}},
$$

where $\left\langle E_{X}\right\rangle$ and $\left\langle B_{X}\right\rangle$ are the spatially averaged dark photon electric and magnetic fields today, which are expected to be comparable to the local field strengths in our galaxy. If the dark photon gauge fields were produced before the matter-radiation equality, i.e., $z_{X}>z_{\mathrm{eq}}=3400$, their cosmological energy density would be constrained by the CMB power spectrum as $\Delta N_{\text {eff }}(X) \lesssim 0.3$ [53], suggesting the local $B_{\mathrm{XT}} \lesssim \mathcal{O}(1) \mu \mathrm{G}$ in our galaxy. On the other hand, if $B_{\mathrm{XT}}$ were produced after the matter-radiation equality (or the recombination), which might be required to avoid a significant distortion of CMB [see (34)], this bound can be relaxed and $B_{\mathrm{XT}}$ significantly bigger than $1 \mu \mathrm{G}$ is allowed. As we will see in the next subsection, if generated by an ultralight ALP well after the matter-radiation 
equality, the produced $B_{\mathrm{XT}}$ can be even as large as $\sim 10 \mu \mathrm{G}$ without any conflict with the known observational constraints.

The photon-ALP-dark photon oscillations induced by $g_{a \gamma \gamma^{\prime}}$ can result in spectral irregularities of other gamma-ray sources, and non-observation of such irregularity may provide an upper bound on $g_{a \gamma \gamma^{\prime}}$. Indeed it has been noticed recently that the Fermi-LAT and MAGIC data of gammarays from NGC1275 in the center of the Perseus cluster

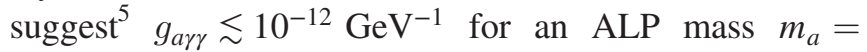
$\mathcal{O}\left(10^{-10}-10^{-9}\right)$ eV [10]. However such consideration cannot be applied to our case because the possible spectral irregularity caused by $g_{a y \gamma}$ severely depends on the detailed profile (including the directions) of the background dark photon gauge fields ${ }^{6}$ along the line of sight between the earth and NGC 1275, on which we do not have any information.

\section{Generation of the background dark photon gauge fields}

A key ingredient of our scenario is the background dark photon gauge fields $E_{X}$ and/or $B_{X}$ which are either comparable to or even stronger than the galactic magnetic fields $B \sim 1 \mu \mathrm{G}$. As there is no reason that the dark photon gauge fields are particularly strong in our galaxy, it is expected that a comparable size of dark photon gauge fields exist over the entire universe. An attractive mechanism to generate such cosmological background gauge fields is to amplify the vacuum fluctuations through the tachyonic instability caused by an evolving axionlike field [30-38]. As was noticed in the previous subsection, to avoid a resonant conversion of CMB to ALP in the early universe [13-15], which would result in a dangerous distortion of $\mathrm{CMB}$ spectrum, those dark photon gauge fields should be generated at a late time with the corresponding redshift factor $z_{X}<z_{\text {res }} \simeq$ few $\times 10^{3}$ where $m_{\gamma}\left(z_{\text {res }}\right)=m_{a}=\mathcal{O}\left(10^{-9}\right) \mathrm{eV}$. If one wishes to have $E_{X}$ and/or $B_{X}$ significantly stronger than $1 \mu \mathrm{G}$, then the background dark photon gauge fields need to be generated well after the matter-radiation equality, e.g., at $z_{X}<1000$, to avoid the bound on the dark photon energy density from the CMB power spectrum [53]. In the following, we present an explicit model for such a late generation of the cosmological background dark photon gauge fields, involving an additional ultralight ALP $\phi$ whose low energy effective Lagrangian is given by

\footnotetext{
${ }^{5} \mathrm{~A}$ previous study [9] indicates that $g_{a \gamma \gamma}=\mathcal{O}\left(10^{-10}\right) \mathrm{GeV}^{-1}$ for $m_{a}=\mathcal{O}\left(10^{-9}\right) \mathrm{eV}$ is allowed by the same Fermi-LAT data on the gamma-rays from NGC 1275, and therefore the result of [10] should be taken with some caution.

${ }^{6}$ It depends also on the profile of ordinary magnetic fields inside the Perseus cluster.
}

$$
\mathcal{L}_{\phi}=\frac{1}{2} \partial^{\mu} \phi \partial_{\mu} \phi-\frac{1}{2} m_{\phi}^{2} \phi^{2}-\frac{1}{4} g_{\phi \gamma^{\prime} \gamma^{\prime}} \phi X_{\mu \nu} \tilde{X}^{\mu \nu} .
$$

In the early universe with the Hubble expansion rate $H \gg m_{\phi}, \phi$ is frozen at its initial value $\phi_{i}$. Around the time when $H$ becomes comparable to $m_{\phi}$, more specifically at $t=t_{\mathrm{osc}}$ when $3 H\left(t_{\mathrm{osc}}\right) \simeq m_{\phi}$, the ultralight ALP $\phi$ begins to oscillate, and evolves for a while as follows

$$
\phi(t) \simeq\left(R(t) / R\left(t_{\mathrm{osc}}\right)\right)^{-3 / 2} \phi_{i} \cos \left(m_{\phi}\left(t-t_{\mathrm{osc}}\right)\right),
$$

where $R(t)$ is the scale factor in the spacetime metric of the expanding universe:

$$
d s^{2}=d t^{2}-R^{2}(t) d \vec{x}^{2}=R^{2}(\tau)\left(d \tau^{2}-d \vec{x}^{2}\right) .
$$

Then the equation of motion for the dark photon field in the momentum space is given by

$$
X_{k \pm}^{\prime \prime}+k\left(k \mp g_{\phi \gamma^{\prime} \gamma^{\prime}} \phi^{\prime}\right) X_{k \pm}=0
$$

where the prime represents the derivative with respect to the conformal time $\tau$, and the subscripts $k$ and \pm denote the comoving momentum and the helicity, respectively. This shows that in the oscillating background $\phi$, one of the helicity states of $X_{k}$ experiences a tachyonic instability for certain range of $k$. The vacuum fluctuations of $X_{k}$ in this range of $k$ are exponentially amplified to be a stochastic classical field [30-38].

In order for this amplification mechanism to be efficient enough in the expanding universe, the ALP coupling $g_{\phi \gamma^{\prime} \gamma^{\prime}}$ times the ALP initial value $\phi_{i}$ needs to be large enough, for instance $g_{X X} \equiv g_{\phi \gamma^{\prime} \gamma^{\prime}} \phi_{i} \gtrsim 70$ to generate $E_{X}$ and/or $B_{X} \gtrsim 1 \mu \mathrm{G}$ at a late time with $z_{X}<1000$. If the amplification mechanism is efficient enough, the produced dark photon gauge fields can affect significantly the evolution of the ALP field $\phi$, which should be taken into account to compute the field strength of the produced dark photon gauge field and also the residual relic energy density of $\phi$ [32-34]. As was demonstrated in the recent works [33,34], this can be achieved by a lattice calculation of the cosmological evolution of ALP and $U(1)$ gauge field in the expanding universe. In order to see explicitly that our mechanism can yield the desired background dark photon gauge fields, we performed (following [33-35]) such a lattice calculation of the present values of the spatially averaged dark photon gauge field strength and the relic mass density of $\phi$ for the parameter region

$m_{\phi} \lesssim 10^{-27} \mathrm{eV}, \quad 100 \lesssim g_{X X} \equiv g_{\phi \gamma^{\prime} \gamma^{\prime}} \phi_{i} \lesssim 200$

We then find 


$$
\begin{gathered}
\left\langle B_{X}\right\rangle\left(\simeq\left\langle E_{X}\right\rangle\right) \simeq(1.2-2) \times\left(\frac{m_{\phi}}{10^{-29} \mathrm{eV}}\right)^{-1 / 3}\left(\frac{\phi_{i}}{10^{17} \mathrm{GeV}}\right) \mu \mathrm{G} \\
\Omega_{\phi} h^{2} \simeq 10^{-4}\left(\frac{\phi_{i}}{10^{17} \mathrm{GeV}}\right)^{2}
\end{gathered}
$$

where the smaller (larger) field strength is obtained for $g_{X X}=100$ (200). We find also that the redshift factor $z_{X}$ at the time $\left(t_{X}\right)$ when the dark photon gauge fields are produced and the present coherent length $\lambda_{X}$ of the produced dark photon gauge fields are determined by $m_{\phi}$ as

$$
\begin{aligned}
\lambda_{X} & \simeq 60\left(\frac{m_{\phi}}{10^{-29} \mathrm{eV}}\right)^{-1 / 3} \mathrm{Mpc}, \\
1+z_{X} & =\frac{R\left(t_{0}\right)}{R\left(t_{X}\right)} \simeq 50\left(\frac{m_{\phi}}{10^{-29} \mathrm{eV}}\right)^{2 / 3} .
\end{aligned}
$$

To explain the gamma-ray spectral modulations noticed in [7] and [8] through the photon-ALP-dark photon oscillations, we need a background dark photon gauge field $B_{\mathrm{XT}} \gtrsim 1 \mu \mathrm{G}$ in our galaxy, where $\vec{B}_{\mathrm{XT}}=\left\langle\vec{B}_{X}\right\rangle-$ $\hat{k}\left(\hat{k} \cdot\left\langle\vec{B}_{X}\right\rangle\right)-\hat{k} \times\left\langle\vec{E}_{X}\right\rangle$. As noticed in the previous subsection, those dark photon gauge fields should be produced at a late time with the redshift $z_{X}<10^{3}$ to avoid a dangerous distortion of CMB [13-15], as well as the constraint on dark radiation from the $\mathrm{CMB}$ power spectrum [53]. Our result (43) shows that such a late production is indeed realized for the ALP mass $m_{\phi}<10^{-27} \mathrm{eV}$.

Looking into the details of the scenario, there could be additional constraints on our scheme, particularly on the relic energy densities of $\phi$ and $X$. Possible constraints on the relic abundance of $\phi$ were summarized in the recent report [54]. According to [54], for the ALP mass range $10^{-33} \mathrm{eV} \ll m_{\phi}<10^{-27} \mathrm{eV}$ which is relevant for us, the most stringent constraint on the energy density $\rho_{\phi}$ comes from the analysis of CMB power spectra including the effect of CMB lensing [55], which leads to the bound on the relic energy density of $\phi$ today:

$$
\left(\Omega_{\phi} h^{2}\right)_{g_{X X}=0} \lesssim 2.7 \times 10^{-3}
$$

for $m_{\phi}=\mathcal{O}\left(10^{-30}-10^{-27}\right) \mathrm{eV}$. Note that this has been derived for the case that there is no conversion of $\rho_{\phi}$ to $\rho_{X}$, and therefore corresponds to the bound for the case $g_{X X}=0$ in our notation.

Yet the above bound can be used to see if our scenario with $100 \lesssim g_{X X} \lesssim 200$ can be compatible with the CMB data. For this, let us briefly examine the evolution of $\rho_{\phi}$ and $\rho_{X}$ in our scenario. Before the exponential amplification of $X$, but after the oscillation of $\phi$, i.e., during the period with

$$
R\left(t_{\mathrm{osc}}\right)<R(t)<R\left(t_{X}\right)
$$

$\rho_{X}$ is negligibly small and therefore

$$
\left(\rho_{\phi}(t)+\rho_{X}(t)\right)_{100 \lesssim g_{X X} \lesssim 200} \simeq \rho_{\phi}(t)_{g_{X X}=0} .
$$

Over a certain period right after the amplification of $X$, e.g., for

$$
R\left(t_{X}\right)<R(t)<3 R\left(t_{X}\right),
$$

both $\rho_{\phi}$ and $\rho_{X}$ show a transient behavior due to the violent amplification of $X$ and the strong backreaction from the amplified $X$. Yet their total energy density is bounded as

$$
\left(\rho_{\phi}(t)+\rho_{X}(t)\right)_{100 \lesssim g_{X X} \lesssim 200} \lesssim \rho_{\phi}(t)_{g_{X X}=0}
$$

since $\rho_{X} \propto R^{-4}$ is more rapidly redshifted than $\rho_{\phi} \propto R^{-3}$. In the final stage with

$$
R(t) \gtrsim 3 R\left(t_{X}\right),
$$

our lattice calculation indicates that

$\left(\rho_{\phi}(t)+\rho_{X}(t)\right)_{100 \lesssim g_{X X} \lesssim 200} \simeq \rho_{\phi}(t)_{g_{X X}=0}\left(c_{1}+c_{2} \frac{R\left(t_{X}\right)}{R(t)}\right)$,

where $c_{1} \simeq 0.3$ and $c_{2} \simeq 1-2$. In summary, $\quad\left(\rho_{\phi}(t)+\right.$ $\left.\rho_{X}(t)\right)_{100 \lesssim g_{X X} \lesssim 200}$ is always bounded by $\rho_{\phi}(t)_{g_{X X}=0}$ and its evolution is similar to that of $\rho_{\phi}(t)_{g_{X X}=0}$ except for a short transient period near $t_{X}$. From this, barring an uncertainty of factor few, we can draw a conclusion that our scheme for model parameters satisfying the bound (44) is compatible with the CMB data. Using (50), we can translate (44) to the bound on $\rho_{\phi}$ today for the case of $100 \lesssim g_{X X} \lesssim 200$, which results in the bound

$$
\left(\Omega_{\phi} h^{2}\right)_{100 \lesssim g_{X X} \lesssim 200} \lesssim 2.7 c_{1} \times 10^{-3} \simeq 8 \times 10^{-4}
$$

for $m_{\phi}=\mathcal{O}\left(10^{-30}-10^{-27}\right) \mathrm{eV}$.

We can now combine our results (42) and (43) with the CMB bound (51). We then find that our mechanism can successfully produce the desired $B_{\mathrm{XT}} \simeq 1-10 \mu \mathrm{G}$, while satisfying the known observational constraints, for the ALP mass $m_{\phi}=\mathcal{O}\left(10^{-30}-10^{-29}\right) \mathrm{eV}$ and the initial ALP misalignment $\phi_{i}=\mathcal{O}\left(10^{17}\right) \mathrm{GeV}$.

\section{CONCLUSION}

In this paper, we proposed a scenario that the spectral irregularities of gamma-rays from some galactic pulsars and supernova remnants, which were recently noticed in [7] and [8], are explained by means of an axionlike particle (ALP) with a mass $m_{a}=\mathcal{O}\left(10^{-9}\right) \mathrm{eV}$ and the coupling $g_{a \gamma \gamma^{\prime}} a X_{\mu \nu} \tilde{F}^{\mu \nu}$ in the range $g_{a \gamma \gamma^{\prime}}=\mathcal{O}\left(10^{-11}-10^{-10}\right) \mathrm{GeV}^{-1}$, 
where $F_{\mu \nu}$ is the ordinary electromagnetic field and $X_{\mu \nu}$ is the field strength of a massless dark photon. A key ingredient of our scenario is the presence of cosmological background dark photon gauge fields, $E_{X} \sim B_{X} \gtrsim 1 \mu \mathrm{G}$, which can be successfully generated by an additional ultralight ALP $\phi$ with $m_{\phi}=\mathcal{O}\left(10^{-30}-10^{-29}\right) \mathrm{eV}$ and the initial field value $\phi_{i}=\mathcal{O}\left(10^{17}\right) \mathrm{GeV}$, whose late coherent oscillations cause a tachyonic instability of the dark photon gauge fields and amplify their vacuum fluctuations to the desired strength. Contrary to the scenario based on the conventional ALP coupling $g_{a \gamma \gamma} a F_{\mu \nu} \tilde{F}^{\mu \nu}$, which was explored in [7] and [8], our scenario can be compatible with the existing observational constraints.

\section{ACKNOWLEDGMENTS}

This work was supported by IBS under the project code, IBS-R018-D1. We thank M. Reece for drawing our attention to [7] and [8], and K. Kamada for useful discussions. We are particularly grateful to N. Kitajima, T. Sekiguchi, and S. Kim for helpful discussions on the lattice simulation. We also thank C. S. Shin and K. Kadota for useful comments and suggestions.
[1] R. Essig et al., Working Group Report: New Light Weakly Coupled Particles, in Proceedings, 2013 Community Summer Study on the Future of U.S. Particle Physics: Snowmass on the Mississippi (CSS2013): Minneapolis, MN, USA (2013) [arXiv:1311.0029].

[2] G. Raffelt and L. Stodolsky, Mixing of the photon with low mass particles, Phys. Rev. D 37, 1237 (1988).

[3] D. Wouters and P. Brun, Constraints on axion-like particles from x-ray observations of the hydra Galaxy cluster, Astrophys. J. 772, 44 (2013).

[4] M. Berg, J. P. Conlon, F. Day, N. Jennings, S. Krippendorf, A. J. Powell, and M. Rummel, Constraints on axion-like particles from x-ray observations of NGC1275, Astrophys. J. 847, 101 (2017).

[5] J. P. Conlon, F. Day, N. Jennings, S. Krippendorf, and M. Rummel, Constraints on axion-like particles from nonobservation of spectral modulations for x-ray point sources, J. Cosmol. Astropart. Phys. 07 (2017) 005.

[6] M. C. D. Marsh, H. R. Russell, A. C. Fabian, B. P. McNamara, P. Nulsen, and C. S. Reynolds, A new bound on axion-like particles, J. Cosmol. Astropart. Phys. 12 (2017) 036.

[7] J. Majumdar, F. Calore, and D. Horns, Search for gammaray spectral modulations in Galactic pulsars, J. Cosmol. Astropart. Phys. 04 (2018) 048.

[8] Z.-Q. Xia, C. Zhang, Y.-F. Liang, L. Feng, Q. Yuan, Y.-Z. Fan, and J. Wu, Searching for spectral oscillations due to photon-axionlike particle conversion using the Fermi-LAT observations of bright supernova remnants, Phys. Rev. D 97, 063003 (2018).

[9] M. Ajello et al. (Fermi-LAT Collaboration), Search for Spectral Irregularities due to Photon-Axionlike-Particle Oscillations with the Fermi Large Area Telescope, Phys. Rev. Lett. 116, 161101 (2016).

[10] D. Malyshev, A. Neronov, D. Semikoz, A. Santangelo, and J. Jochum, Improved limit on axion-like particles from $\gamma$-ray data on Perseus cluster, arXiv:1805.04388 [Phys. Rev. D (to be published)].

[11] A. Abramowski et al. (H.E.S.S. Collaboration), Constraints on axionlike particles with H.E.S.S. from the irregularity of the PKS 2155-304 energy spectrum, Phys. Rev. D 88, 102003 (2013).

[12] A. Mirizzi, G. G. Raffelt, and P. D. Serpico, Photon-axion conversion as a mechanism for supernova dimming: Limits from CMB spectral distortion, Phys. Rev. D 72, 023501 (2005).

[13] A. Mirizzi, J. Redondo, and G. Sigl, Constraining resonant photon-axion conversions in the Early Universe, J. Cosmol. Astropart. Phys. 08 (2009) 001.

[14] H. Tashiro, J. Silk, and D. J. E. Marsh, Constraints on primordial magnetic fields from $\mathrm{CMB}$ distortions in the axiverse, Phys. Rev. D 88, 125024 (2013).

[15] S. Mukherjee, R. Khatri, and B. D. Wandelt, Polarized anisotropic spectral distortions of the CMB: Galactic and extragalactic constraints on photon-axion conversion, J. Cosmol. Astropart. Phys. 04 (2018) 045.

[16] M. Simet, D. Hooper, and P. D. Serpico, The Milky Way as a Kiloparsec-scale axionscope, Phys. Rev. D 77, 063001 (2008).

[17] A. De Angelis, M. Roncadelli, and O. Mansutti, Evidence for a new light spin-zero boson from cosmological Gammaray propagation?, Phys. Rev. D 76, 121301 (2007).

[18] A. De Angelis, O. Mansutti, M. Persic, and M. Roncadelli, Photon propagation and the VHE gamma-ray spectra of blazars: How transparent is really the Universe?, Mon. Not. R. Astron. Soc. 394, L21 (2009).

[19] M. A. Sanchez-Conde, D. Paneque, E. Bloom, F. Prada, and A. Dominguez, Hints of the existence of axion-like-particles from the gamma-ray spectra of cosmological sources, Phys. Rev. D 79, 123511 (2009).

[20] A. Avgoustidis, C. Burrage, J. Redondo, L. Verde, and R. Jimenez, Constraints on cosmic opacity and beyond the standard model physics from cosmological distance measurements, J. Cosmol. Astropart. Phys. 10 (2010) 024.

[21] A. Dominguez, M. A. Sanchez-Conde, and F. Prada, Axionlike particle imprint in cosmological very-high-energy sources, J. Cosmol. Astropart. Phys. 11 (2011) 020.

[22] D. Horns, L. Maccione, M. Meyer, A. Mirizzi, D. Montanino, and M. Roncadelli, Hardening of TeV gamma spectrum of AGNs in galaxy clusters by conversions of photons into axion-like particles, Phys. Rev. D 86, 075024 (2012). 
[23] G. Galanti, M. Roncadelli, A. De Angelis, and G. F. Bignami, Advantages of axion-like particles for the description of very-high-energy blazar spectra, arXiv:1503.04436.

[24] P. Tiwari, Constraining axionlike particles using the distance-duality relation, Phys. Rev. D 95, 023005 (2017).

[25] K. Kohri and H. Kodama, Axion-like particles and recent observations of the cosmic infrared background radiation, Phys. Rev. D 96, 051701 (2017).

[26] V. Anastassopoulos et al. (CAST Collaboration), New CAST limit on the axion-photon interaction, Nat. Phys. 13, 584 (2017).

[27] A. Payez, C. Evoli, T. Fischer, M. Giannotti, A. Mirizzi, and A. Ringwald, Revisiting the SN1987A gamma-ray limit on ultralight axion-like particles, J. Cosmol. Astropart. Phys. 02 (2015) 006.

[28] K. Kaneta, H.-S. Lee, and S. Yun, Portal Connecting Dark Photons and Axions, Phys. Rev. Lett. 118, 101802 (2017).

[29] K. Kaneta, H.-S. Lee, and S. Yun, Dark photon relic dark matter production through the dark axion portal, Phys. Rev. D 95, 115032 (2017).

[30] M. M. Anber and L. Sorbo, Naturally inflating on steep potentials through electromagnetic dissipation, Phys. Rev. D 81, 043534 (2010).

[31] N. Barnaby, E. Pajer, and M. Peloso, Gauge field production in axion inflation: Consequences for monodromy, nonGaussianity in the CMB, and gravitational waves at interferometers, Phys. Rev. D 85, 023525 (2012).

[32] P. Agrawal, G. Marques-Tavares, and W. Xue, Opening up the QCD axion window, J. High Energy Phys. 03 (2018) 049.

[33] N. Kitajima, T. Sekiguchi, and F. Takahashi, Cosmological abundance of the QCD axion coupled to hidden photons, Phys. Lett. B 781, 684 (2018).

[34] K. Choi, H. Kim, and T. Sekiguchi, Late-Time Magnetogenesis Driven by Axionlike Particle Dark Matter and a Dark Photon, Phys. Rev. Lett. 121, 031102 (2018).

[35] P. Agrawal, N. Kitajima, M. Reece, T. Sekiguchi, and F. Takahashi, Relic abundance of dark photon dark matter, Phys. Lett. B 801, 135136 (2020).

[36] J. A. Dror, K. Harigaya, and V. Narayan, Parametric resonance production of ultralight vector dark matter, Phys. Rev. D 99, 035036 (2019).

[37] R. T. Co, A. Pierce, Z. Zhang, and Y. Zhao, Dark photon dark matter produced by axion oscillations, Phys. Rev. D 99, 075002 (2019).

[38] M. Bastero-Gil, J. Santiago, L. Ubaldi, and R. VegaMorales, Vector dark matter production at the end of inflation, J. Cosmol. Astropart. Phys. 04 (2019) 015.

[39] G. G. Raffelt, Stars as Laboratories for Fundamental Physics (University of Chicago Press, 1996).
[40] K. Choi, H. Kim, and S. Yun, Natural inflation with multiple sub-Planckian axions, Phys. Rev. D 90, 023545 (2014).

[41] D. E. Kaplan and R. Rattazzi, Large field excursions and approximate discrete symmetries from a clockwork axion, Phys. Rev. D 93, 085007 (2016).

[42] K. Choi and S. H. Im, Realizing the relaxion from multiple axions and its UV completion with high scale supersymmetry, J. High Energy Phys. 01 (2016) 149.

[43] G. F. Giudice and M. McCullough, A clockwork theory, J. High Energy Phys. 02 (2017) 036.

[44] T. Higaki, K. S. Jeong, N. Kitajima, and F. Takahashi, The QCD axion from aligned axions and Diphoton excess, Phys. Lett. B 755, 13 (2016).

[45] P. Agrawal, J. Fan, M. Reece, and L.-T. Wang, Experimental targets for photon couplings of the QCD axion, J. High Energy Phys. 02 (2018) 006.

[46] M. Farina, D. Pappadopulo, F. Rompineve, and A. Tesi, The photo-philic QCD axion, J. High Energy Phys. 01 (2017) 095.

[47] R. Jansson and G. R. Farrar, A new model of the Galactic magnetic field, Astrophys. J. 757, 14 (2012).

[48] G. G. Raffelt, Core mass at the Helium flash from observations and a new bound on neutrino electromagnetic properties, Astrophys. J. 365, 559 (1990).

[49] E. L. Chupp, W. T. Vestrand, and C. Reppin, Experimental Limits on the Radiative Decay of SN1987A Neutrinos, Phys. Rev. Lett. 62, 505 (1989).

[50] T. Fischer, S. C. Whitehouse, A. Mezzacappa, F. K. Thielemann, and M. Liebendorfer, Protoneutron star evolution and the neutrino driven wind in general relativistic neutrino radiation hydrodynamics simulations, Astron. Astrophys. 517, A80 (2010).

[51] A.-C. Davis, M. Lilley, and O. Tornkvist, Relaxing the bounds on primordial magnetic seed fields, Phys. Rev. D 60, 021301 (1999).

[52] D. J. Fixsen, E. S. Cheng, J. M. Gales, J. C. Mather, R. A. Shafer, and E. L. Wright, The cosmic microwave background spectrum from the full COBE* FIRAS data set, Astrophys. J. 473, 576 (1996).

[53] N. Aghanim et al. (Planck Collaboration), Planck 2018 results. VI. Cosmological parameters, arXiv:1807.06209.

[54] D. Grin, M. A. Amin, V. Gluscevic, R. Hlǒzek, D. J. E. Marsh, V. Poulin, C. Prescod-Weinstein, and T. L. Smith, Gravitational probes of ultra-light axions, arXiv:1904 .09003 .

[55] R. Hlozek, D. J. E. Marsh, and D. Grin, Using the full power of the cosmic microwave background to probe axion dark matter, Mon. Not. R. Astron. Soc. 476, 3063 (2018). 